\title{
LA REPRODUCCIÓN DE LA DESIGUALDAD INTERGENERACIONAL Y NUEVAS FORMAS DE EXCLUSIÓN SOCIAL PRODUCTO DEL DISEÑO POLÍTICO DEL SISTEMA EDUCACIONAL CHILENO ${ }^{1}$
}

\author{
Sebastián Donoso Díaz
}

\begin{abstract}
O desenho político do sistema social chileno tem sido incapaz de solucionar os problemas endêmicos de desigualdade social que o caracterizam, evidenciando, com isso, uma crise profunda não somente do sistema educacional, mas também do sistema social e econômico, o que demanda um desenho sistêmico diferente, que não se vislumbra ainda como factível. Apesar dos esforços despendidos, os resultados educacionais evidenciam um forte vínculo com a origem social e econômica das famílias, ou seja: as diferenças de capital social e cultural inicial não são atenuadas, corrigidas nem revertidas de forma importante pelo sistema educacional regular. $\mathrm{O}$ problema reside no fato de que a educação apresenta padrões muito desiguais entre os diversos estratos sociais, de modo que, mesmo apresentando semelhanças formais, no fundo, as diferenças são chaves e respondem a uma acumulação de "vantagens e desvantagens", segundo o caso, que finalmente resultam numa reprodução da desigualdade social intergeracional, só que devidamente legitimada. Esse círculo reprodutor da desigualdade é a nova forma como se apresenta o problema, que já não é de "excluídos e incluídos”, mas de novas formas de inclusão ou exclusão complexas, mas igualmente eficientes para manter as diferenças intergeracionais. $O$ fracasso do atual desenho do sistema educacional e social implica visualizar mudanças que requerem uma transformação de magnitude na concepção do Estado, o que se estima ser complexo, pois, de certa forma, é negar-se a si próprio como Estado, para dar um passo em direção a um novo pacto social. PALAVRAS-CHAvE: desigualdade social, sistema educacional chileno, capital social e cultural.
\end{abstract}

\section{INTRODUCCIÓN}

El trabajo analiza la crisis del diseño político del sistema educacional chileno. El concepto crisis ha sido utilizado recurrentemente y, a veces, con sentido muy diferente. Para efectos del texto, se le entiende como la presencia de dificultades severas que comprometen estratégicamente la existencia del objeto en análisis en los términos actuales, cuyo desenlace puede significar su estancamiento como también su progreso. En razón de ello, la crisis del diseño político del sistema educacional, para el caso chileno, reside en que se ha llegado a un punto complejo para el desarrollo del sistema educacional, por cuanto algunas de sus funciones políticas claves en materia de fomento de la cohesión y de legitimación del orden social (en el sentido planteado por Weber [1969 ] y Habermas, [1975]) se encuentran seve-

* Doutor em Educação pela Universidade Academia de Humanismo Cristiano (Chile). Professor do Instituto de Investigação e Desenvolvimento Educacional da Universidade de Talca, Chile.

2 Norte 685, Talca, región del Maule, Chile.sdonoso@utalca.cl

${ }^{1}$ Este artículo es parte del Proyecto de investigación CONICYT- PBCT PSD 60. ramente comprometidas, producto de los grandes problemas que enfrenta la sociedad chilena evidenciados en la fuerte reproducción de la desigualdad intergeneracional y la incubación de nuevas formas de exclusión social que operan con formatos diferentes a los usualmente conocidos.

La crisis del diseño político del sistema educativo chileno se ha tornado estratégica, pues, durante casi dos décadas, los gobiernos democráticos han buscado compatibilizar dos racionales asentados en fundamentos y criterios de operación que han mostrado su contradicción: Estado subsidiario versus Estado solidario, tras un largo proceso político seguido en el período de retorno a la democracia, que extremó las semejanzas y minimizó las diferencias entre ambas perspectivas. El camino a seguir parece cada vez más estrecho, difuso y próximo a agotarse, requiriendo, para su solución, analizar nuevos diseños políticos para el sistema educacional chileno, al amparo posiblemente de la discusión de un rol diferente del Estado, sustentado en un pacto social diferente con compromisos de nuevo orden. 
El período en análisis (1990-2008) implicó sustentar la provisión de una educación de calidad, inclusiva de la equidad, que generara condiciones ciertas en la población, habilidades, competencias, capacidades y destrezas que les facultaran para reducir, en términos significativos, las brechas intergeneracionales y la exclusión social, aspectos puestos en duda en la actualidad, al tenor de los problemas y conflictos que muestra la sociedad chilena en esta materia.

En función de lo expuesto, la relevancia de la experiencia chilena para la región latinoamericana reside en las lecciones que puedan derivarse de un caso que ha manifestado, por casi tres décadas, la búsqueda de una fuerte coherencia entre las políticas económicas de mercado y las políticas sociales que han buscado - casi en los últimos veinte años - reducir sus fallos, impulsado a las políticas educacionales a desempeñar un rol instrumental clave en este aspecto. Esta misión no es menor si se entiende, como lo señala NEF (1999/2000), que los sucesos de los años 70 y 80 son partícipes de la contrarrevolución neoliberal, fenómeno que no fue propio solamente del ámbito chileno.

\section{EL DISEÑO DEL SISTEMA POLÍTICO Y EDUCACIONAL DE LOS AÑOS 80 Y LAS CAMBIOS DE LOS 90 EN ADELANTE \\ Nuevo rol del Estado y soporte legal y financiero del sistema de mercado en la educación}

Bajo la constante de que, en el período en análisis como también en la mayor parte de la vida republicana del país, el Estado chileno asume una visión unitaria con un fuerte componente presidencialista (Caro, 2003) y que constitucionalmente el Estado de Chile se define como unitario, y su administración como funcional y territorialmente descentralizada o desconcentrada en su caso, características que se han mantenido y adecuado, incluso en la última reforma consti- tucional del año 2005. Asumimos que ambas condiciones, la visión unitaria y un régimen fuertemente presidencialista, son parte del problema que se analiza como también parte de la solución que se esboza en la ponencia

Es conocida la fuerte fractura que experimentó la sociedad chilena a partir del año 1973, iniciándose un régimen dictatorial que, por más de 17 años, generó transformaciones relevantes en todos los ámbitos de la sociedad nacional, muchos de los cuales han persistido después de casi dos décadas de democracia. Algunos elementos han sido constantes en esta etapa en análisis: el primero, el cambio de rol del Estado (1975), de una concepción pro bienestar a una de carácter subsidiario y, el segundo, la incorporación, casi sin restricciones, de la política de mercado en todos los ámbitos de la sociedad (excluida la defensa nacional).

El sector educación fue una de los que más tardíamente se ajustó al modelo de economía de mercado de inspiración neoliberal, por cuanto en el mismo Gobierno existían indecisiones y disputas entre dos corrientes: una ligada a la visión más tradicional del Estado en este campo, con un fuerte rol del sector público en la provisión de educación, y otra que propugnaba decididamente su apertura a la privatización, enfatizando, entre otros aspectos, la incorporación de proveedores privados de educación. Este debate se zanja finalmente a mediados del año 80 (Directivas Presidenciales para la Educación, 1980), imponiéndose la perspectiva privatizadora, lo que derivó en cambios de gran relevancia en las políticas e instrumentos de financiamiento de la educación pública en todos sus niveles. De igual forma, ese año, entra en vigencia la nueva constitución política de la República, que formaliza los cambios y establece las proyecciones del modelo en lo social, económico y político.

En el texto de la nueva constitución, a diferencia de los argumentos precedentes de las cartas constitucionales de los años 1833 y 1925, se instituyen nuevos principios respecto del derecho a la educación expuestos en sus artícu- 
los $\mathrm{n}^{\mathrm{o}} 10$ y 11: "Los padres tienen el derecho preferente y el deber de educar a sus hijos. Corresponderá al Estado otorgar especial protección al ejercicio de este derecho.” A pesar de que reconoce que la "educación básica es obligatoria, debiendo el Estado financiar un sistema gratuito con tal objeto," también establece que "....es deber de la comunidad contribuir... [a su] desarrollo y perfeccionamiento." Sin embargo, el tema central del documento es elevar a la categoría de principio "la libertad de enseñanza".

Esta materia - la libertad de enseñanza ha sido uno de los puntos neurálgicos de los debates sobre el tema, iniciado finalmente de manera pública el año $2006^{3}$ (Consejo Asesor Presidencia, 2006) y que a la fecha siguen siendo uno de los aspectos más controversiales de la nueva propuesta de Ley General de Educación (LGE), que se encuentra en el parlamento próxima a iniciar su debate (Mayo, 2008) y que busca sustituir la Ley Orgánica Constitucional de Enseñanza [LOCE] - aprobada en los últimos días del Gobierno de Pinochet y vigente, con modificaciones a la fecha.

El principio de Libertad de Enseñanza “... habilita el derecho a abrir, organizar y mantener establecimientos educacionales...", no teniendo "otras limitaciones que las impuestas por la moral, las buenas costumbres, el orden público y la seguridad nacional...”. Debe entenderse este principio como el fundamento del proceso privatizador de la educación, inherente al modelo de mercado, que busca garantizar el amplio ingreso y salida de proveedores sin mayores limitaciones (a fin de generar el mercado). $\mathrm{Al}$

${ }^{2}$ En el año 2003, el Gobierno democrático extiende este derecho a la enseñanza secundaria.

${ }^{3}$ En mayo del año 2006 se produce un movimiento de los estudiantes de enseñanza secundaria, denominado "los pingüinos“, que puso en jaque a la autoridad sectorial, demandando cambios de magnitud en el sistema. A raíz de este movimiento se formó un Consejo Asesor que debate estas materias y concluye en un informe con recomendaciones, algunas con amplio consenso y otros sin esta condición. Dos aspectos son neurálgicos en esta materia, y no fueron resueltos por consenso: el conflicto entre el principio de libertad de enseñanza y el de calidad de la enseñanza, y en lo operacional, el derecho a seleccionar o no estudiantes y la posibilidad que las instituciones educativas puedan obtener lucro. respecto y siendo coherente con la concepción ideológica que le sustenta, establece que la "enseñanza reconocida oficialmente no podrá orientarse a propagar tendencia político partidista alguna...” Finalmente, el principio de libertad de enseñanza supone, primero y antes que toda opción, que los "padres tienen el derecho de escoger el establecimiento de enseñanza para sus hijos."

La arquitectura del sistema educacional fue sinérgica con la puesta en vigencia de la nueva constitución, comenzando a funcionar a partir del año 1981, la cual se operacionaliza mediante cinco aspectos claves y, ciertamente, bajo un escenario de fuertes restricciones democráticas que impedía cualquier oposición razonable a estas propuestas. Los aspectos claves son:

- Los establecimientos escolares - que, en su totalidad, pertenecían al Estado nacional-fueron transferidos a los gobiernos locales (Municipios).

- De igual forma, los docentes y personal no docente fueron transferidos a los gobiernos locales, perdiendo un conjunto relevante de beneficios económicos y de carrera profesional que, incluso a la fecha, aún no han recuperado del todo, pese a los reconocidos esfuerzos desplegados por los gobiernos democráticos (Rojas, 1998).

- Se estimuló y facilitó la libre entrada o salida de proveedores privados de educación, mediante los nuevos instrumentos financieros de operación.

- Se cambió el sistema de financiamiento de educación pública, de un modelo sustentado en financiar la oferta a uno orientado a la demanda.

- Ello también implicó una concepción de educación pública diferente a la entonces vigente. Por tal se entenderá - desde entonces a la fecha - todos los establecimientos educacionales que reciben subsidio del Estado independiente de la naturaleza de su propietario.

El período de transición entre 1980-1981 marca un límite relevante para la temática en análisis, por cuanto el año 1980 fue el último en el cual el Estado chileno financió con recursos públicos la oferta y provisión directa de educación, como también por la legitimación constitucio- 
nal de la reorientación del accionar del Estado hacia la subsidiariedad, definida como de prestaciones básicas, incentivos y contribuciones cuando los privados no puedan asumir las tareas. También en materia educacional es un hito relevante debido a que implicó elevar a principio central del sistema educacional la Libertad de Enseñanza, posteriormente consagrada en al LOCE el año 1989 y operacinalizada como el derecho primordial de los padres a elegir la educación de sus hijos, y en lo institucional para el sistema educacional significo asumir la libre entrada y salida de oferentes o demandantes de la educación, en pos de sincronizar el sector educación con la generación de mercados en esta materia. Para el logro de estos objetivos, el cambio del sistema de financiamiento fue el factor crucial que permitió alcanzar estos objetivos en el corto plazo, y se transformó en el motor de la contrarrevolución neoliberal hasta nuestros días.

\section{El objetivo político del nuevo modelo de financiamiento: generación del mercado de la educación pública en Chile}

La necesidad de sincronizar el funcionamiento del sector educación con la economía de mercado fue el principio político clave que impulsó el cambio del modelo de financiamiento del sistema educacional. En razón de ello, de los cinco elementos mencionados (sección 2.1), el aspecto clave corresponde al cambio del sistema de financiamiento de la educación pública, el cual demandó, concomitantemente para su implantación, las restantes condiciones mencionadas.

El cambio del sistema de financiamiento ha sido una de las transformaciones más eficaces impulsadas en el sector educación en los últimos 40 años, por cuanto no solamente incide en los criterios, orientaciones y mecanismos de financiamiento, sino que también tiene impactos relevantes en lo pedagógico, de forma que se trató, finalmente, de una reforma educacional de magnitud, impulsada desde las políticas de financiamiento para el sector. ${ }^{4}$

El proceso sustitutivo del modelo de financiamiento, basado en la oferta por el sustentado en la demanda, no respondió a una discusión pública, ni a un diagnóstico que diera cuenta del fracaso del formato de subsidio a la oferta. La literatura de la época muestra la carencia de estudios empíricos que fundamenten la decisión adoptada (Jofré, 1988). Sus objetivos políticos más evidentes eran dos: consolidar el modelo privado de desarrollo económico, mediante la imposición de un sistema de subsidio basado - efectivamente - en el racional privado, tanto para operadores públicos (municipales) como privados, y favorecer la descentralización de la gestión del Estado, traspasando a los gobiernos subnacionales atribuciones en este plano y, paralelamente, reduciendo su impacto al fortalecer el accionar de los privados.

Complementariamente, el nuevo diseño del sector buscaba fortalecer los objetivos propuestos mediante un mayor control del gasto público, derivado del sistema de asignación de los subsidios, y también, mejorar el rendimiento de los estudiantes mediante el desarrollo y fortalecimiento de la competencia entre los establecimientos públicos y privados, pues supone que, por esta vía, aumentará la eficiencia, proviniendo mejores resultados educacionales.

La complejidad de las tareas que se impulsaron con los cambios de 1981 fue alta, la que se eleva ante el hecho que las autoridades gubernamentales de la época implantan las nuevas medidas de financiamiento de la educación simultáneamente con una fuerte reducción del presupuesto público del sector, que alcanzó al 24\% real entre los años 1982 y 1989 (González, 2003, p.610; Sapelli, 2002, p.285). Esta situación es crucial para comprender parte del deterioro de los resultados educacionales del país que aún persisten, los que suelen subestimarse por los

\footnotetext{
${ }^{4}$ Se enfatiza esta condición pues, al revisarse las reformas políticas de la educación chilena, no siempre es consignada como tal. Sin embargo, precisamente su existencia inadvertida como "reforma expresa" es la que facilitó su expansión a todas las áreas del proceso educativo.
} 
autores proclives a este enfoque, olvidando, entre otros aspectos, que el nuevo sistema de subsidio creó - entre otros efectos - severos problemas de financiamiento del sector municipal, al extremo que, en 1986, tuvo que reajustarse el valor del subsidio en forma relevante para evitar el colapso del sistema (Jofré, 1988).

El criterio central de asignación de los recursos financieros se sustenta en su asociación con la demanda por educación. Este funciona como un subsidio que se entrega al propietario del establecimiento escolar, sea este público (el municipio) o un empresario privado. El monto entregado es la resultante de un valor basal relacionado "el promedio de asistencia diaria a clases del estudiante", empleando para ello el formato de trimestre/móvil (con un tope de 20 días mes). Esta resultante se asocia al valor mes del subsidio, dando la cifra efectiva por estudiante, la cual es entregada por el Ministerio de Educación a los empresarios (públicos y privados). Este sistema de subsidio aplicado en Chile, denominado "vales o vouchers lump sum", ha mostrado ser menos eficiente que otros mecanismo equivalentes, usualmente asociados a condiciones socioeconómicas de la población y al porcentaje de aporte del hogar a educación (Hoxby, 2002).

La educación primaria en Chile asigna por esta vía la mayor parte de los recursos a los empresarios públicos y privados. El subsidio de cada estudiante de educación básica regular (2008) tiene un precio base mes de US \$ 80 y de US \$ 95 para la enseñanza secundaria. En el caso de los Municipios, en la enseñanza básica, no pueden cobrar a los padres valores adicionales (salvo cifras menores que no suman los US \$ $10 \mathrm{al}$ año por estudiante), $y$, en la enseñanza secundaria, sólo pueden hacerlo con el beneplácito de los padres. No es así con los empresarios privados que, cumpliendo determinados procedimientos, optan al sistema de "financiamiento compartido", que les permite incrementar sus ingresos en valores que van entre los US \$ 5 a 20 por alumno mes. ${ }^{5}$

${ }^{5}$ US $\$ 1,0=\$ 450$ pesos chilenos (abril 2008).
Por su parte, el Ministerio entrega a los municipios recursos para mantención de infraestructura escolar, que suelen ser cifras menores. También el Ministerio interviene en algunos establecimientos públicos con programas (destinados a escuelas prioritarias o población focalizada - desertores, etc.) que implican asignación de recursos directos fundamentalmente en textos, materiales, horas de trabajo y asistencia técnica, más que recursos financieros frescos para el municipio. Este proceder se podría catalogar que funciona con el racional de subsidio a la oferta y muestra que existen aún algunos instrumentos que confieren al sistema chileno aplicación de instrumentos mixtos, aunque, al revisarse las cifras que están involucradas, no cabe duda alguna que ello no es así, más del 62\% de los gastos del sector central (Gobierno) son referidos a subvenciones y menos del 7\% se asigna en programas directos (DIPRES, 2003).

El 92\% de todas las transferencias que realiza el Ministerio al sector en estos ítemes son referidas a la escolaridad (asistencia de alumnos); el restante porcentaje se asigna por compensación de ruralidad, desempeño difícil, internado y otra serie de ponderadores que son secundarios o terciarios respecto de los montos involucrados (DIPRES, 2004). De igual forma, hasta lo que va del año 2008 (mayo), existe prácticamente sólo un valor de subsidio para cada nivel de enseñanza, ${ }^{6}$ donde el $93 \%$ del monto total asignado es fijo y el 7\% es variable. Este punto es uno de los temas más complejos de debate y si bien se supone que a partir del año 2008 se aplicará un subsidio adicional. Asociado a las condiciones de mayor pobreza de la población, el reglamento de operación de este proceso no está aún en régimen, por ende no entra en operación. Sin embargo, si bien es un avance hacia una política que responda en mejor medi${ }^{6}$ Se han definido diferentes subsidios: preescolar, enseñanza básica, enseñanza media científico/humanista y enseñanza media técnico/profesional y educación especial. Existen otras bonificaciones por desempeño difícil, ruralidad y el subsidio base para escuelas pequeñas (paga el equivalente a 17 alumnos aunque su matrícula sea menor. 
da a los costos de formación de los estudiantes con menor capital humano, lo cierto es que dista aún de asumir el problema en la integralidad de sus dimensiones.

Una fuente de recursos destinados fundamentalmente a infraestructura y, en menor medida, a equipamiento proviene de fondos regionales (Fondo Nacional de Desarrollo Regional), que son recursos aportados por le gobierno central para que sean administrados por los gobiernos subnacionales. Su monto al año 2003 destinado a educación no superó el 6\% del asignado a subvención. También los Municipios destinan cifras menores para gastos corrientes, fundamentalmente salarios y otros que, en montos totales, no superan el 2,5\% de los recursos destinados centralmente a subvención, pero que, en algunos casos de municipios pequeños, esta cifra es relevante.

Finalmente, en el caso chileno, el aporte privado a educación es de gran significación. Del $7,3 \%$ del PIB aportado a educación (2006), el $46 \%$ es de fondos privados, situación que se explica fundamentalmente por la educación superior; sin embargo, la participación de gastos privados en todos los niveles es un fenómeno cierto y creciente masivo; ya en la enseñanza secundaria, el 56\% de la matricula escolar es privada y, en la educación superior, más de la mitad de las vacantes anuales también lo son (MINEDUC, 2007; ÍNDICES, 2008).

El sistema de financiamiento mediante subsidio ha tenido muchos problemas en su aplicación en Chile. Algunos provienen de la forma cómo fue implantado, otros de su diseño y otros de los supuestos y considerandos que asume. Dependiendo de la naturaleza del problema, en el primer grupo de críticos se sitúan prioritariamente aquellos que difieren radicalmente con la concepción política del tema; en el segundo grupo, aunque comparten el modelo, tienen reparos técnicos; mientras que, en el tercer grupo, se encuentran los que tienen reparos técnicos porque no comparten el modelo.

Las críticas a la legitimidad del modelo y su implantación se refieren al origen viciado de su diseño y aplicación en plena dictadura y sin posibilidades de participación social. Concomitantemente, aluden al afán privatizador de todas las medidas propuestas, incluyendo la descentralización de la gestión del Ministerio de Educación y el traspaso de establecimientos y docentes a dependencias de los gobiernos locales.

Los puntos en desacuerdo en el plano técnico entre quienes comparten los fundamentos y principios operativos del subsidio y sus mecanismos de aplicación residen básicamente en que, al establecer un sistema de subsidio de precio único con pago similar para todos (como es el caso chileno), se auxilia más a quien tiene mayor capital humano inicial, es decir genera un efecto perverso que favorece a los que tienen costos formativos menores, perjudicando, por esta vía, a los que poseen condiciones de mayor pobreza (Sapelli, 2002, p.287).

Lo interesante del caso chileno es que, sabiéndose desde hace tiempo estos efectos, los sistemas de corrección empleados, como son los programas ofertados a escuelas que atienden a la población e bajos ingresos, han sido insuficientes para revertir los efectos negativos del modelo implantado y, tras casi dos décadas de democracia, no se puede apelar a la "herencia no deseada de la dictadura". Ha transcurrido un tiempo razonable para su corrección. ${ }^{7}$ Ello ha dado origen a diversos submercados educativos con calidad de educación muy diferente, no comparables, que conforman lo que se ha denominado los nuevos sistemas de exclusión social y de reproducción de la desigualdad.

Este es quizás uno de los principales problemas que generó la aplicación de esta política de financiamiento, de que derivó finalmente un sistema altamente segmentado socialmente, con diferenciales de calidad muy importantes. Entre los distintos autores, el consenso más alto en esta

En la primera mitad de la década de los 90, se generó un subsidio rural de mejor valor, así como otro para escuelas pequeñas (que se paga un equivalente a 18 estudiantes aunque no los tenga) y otro por desempeño difícil. Se trató de subsidios que implicaron una cobertura baja respecto del sistema total. 
materia proviene del convencimiento que el precio del subsidio fue la determinante fundamental para la generación de un sistema tan segmentado socialmente. Pero para quienes el sistema de subsidio no es un "dogma de fe", el problema no reside sólo en el valor del subsidio, sino en su forma de cálculo y, más profundamente, en el sistema mismo de subsidio de prestaciones básicas que se ha impuesto "urbi et orbi” en la educación chilena.

Cuesta entender que sólo a partir del 2008 se haya establecido un valor de subsidio diferente para un segmento de la población escolar más pobre, ya que había sido denunciado por autores de todas las corrientes de opinión como un tema capital que debía repararse. La actual propuesta de "subsidio preferencial" otorga un $30 \%$ adicional de recursos por estudiantes (US \$ 40) a los establecimientos que incorporan al menos el $15 \%$ de población perteneciente a los segmentos sociales más pobres. De esta forma, el sistema hoy debería tener dos niveles, el valor regular y el preferencial. Según los partidarios del modelo, el tema "precio" era finalmente el que desvirtuaba el sistema y generó la estratificación señalada. Es decir, con precios ajustados al costo de la formación por nivel socioeconómico, ese problema no debió haberse generado. Para los críticos, es una consecuencia inevitable del sistema de mercado y los subsidios diferenciados corrigen sólo parcialmente esta situación.

En otra materia, los partidarios del modelo de financiamiento a la demanda sostienen que la existencia de subsidios a la oferta, es decir, algunos programas que aportan recursos preferentemente a los establecimientos municipales, sería un impuesto al traslado de los estudiantes, pues castiga a quienes se cambian de establecimiento escolar (es decir, al traslado hacia el sector privado). Los hechos han demostrado que este fenómeno no sigue "la crítica señalada"; por el contrario, la revisión de la evolución de la matrícula de estudiantes por tipo de establecimiento escolar (Municipal/particular subvencionado) da cuenta de cambios de magnitud ocurridos entre los años 1980 y 2007. En síntesis, el sector municipal tenía el 80\% de matrícula de niños el año inicial señalado y pasa a tener el 50\% el 2007, compartiendo en igual proporción con el sector subvencionado (MINEDUC, 2008).

El tema expuesto evidencia algunos de los problemas derivados de la implantación del modelo. Como fue señalado, hay la segmentación del sector educación en varios cuasimercados (Almonacid y otros, 2008), que se transforman en segmentos con escasa interacción en vez de mercados dinámicos. Una muestra de lo señalado es que la educación rural, que, en el caso chileno, concentra a los grupos sociales de menores ingresos, es atendida en un $85 \%$ por los establecimientos municipales, teniendo los privados una presencia exigua (MINEDUC, 2007). De igual forma, los resultados de las pruebas de medición de aprendizajes (SIMCE) de aplicación censal nacional para los grados 4, 8 y 12 han demostrado sistemáticamente, desde el año 1988 en adelante, con sistemas menos o más sofisticados de construcción de ítemes y de equivalencia de preguntas (equating), que la población que pertenece a los dos quintiles socioeconómicamente más precarios y que asiste a establecimientos escolares municipales tiene mejores resultados en las pruebas que los particulares que reciben subsidio del Estado (MINEDUC, 2007a). También estos resultados han mantenido una estrecha relación con el capital social y económico de los estudiantes y sus familias y sólo marginalmente con el tipo de estableciendo; de allí que la determinante socioeconómica siga siendo el gran factor explicativo de los resultados educacionales en todos los niveles del sistema.

El problema de este factor es su gran peso para incidir en los resultados finales, es decir, el sistema educacional no reduce en grado importante las diferencias "de cuna" de los estudiantes (Donoso; Hawes, 2002; Hsieh; Urquiola, 2004).

Las señales que por larga data ha venido emitiendo el sistema es que esta modalidad de financiamiento no resulta positiva para quienes viven en situación de mayor pobreza. El modelo presume que a mayor asistencia de los estudiantes a clases, se genera más aprendizaje y por ende 
mayor calidad. Sin embargo, su aplicación omite una serie de considerandos claves para comprender los resultados alcanzados en Chile, en este período de tiempo, que distan de lo esperado:

- las diferencias de capital humano inicial de la población escolar;

- los recursos asociados a la producción de una educación de calidad según las diversas dotaciones de capital inicial de la población;

- acceso y capacidad de comprensión de la información necesaria para adoptar las decisiones de parte de las familias (simetría de información);

- dotación de capital humano, infraestructura y equipamiento de las instituciones educacionales;

- oferta territorial uniforme de educación de calidad y mecanismos técnicos adecuados para implantar y controlar el sistema de asignación de recursos (Donoso y Schmal: 2008).

La evidencia del caso chileno es suficientemente fuerte para sustentar que el mercado no ha regulado por la vía de la demanda los principales fallos, ratificados en gran parte por las mejoras que se busca implantar consensudamente el año 2008, cuya eventual aplicación está siendo crecientemente puesta en duda, debido a la necesidad de repensar esta situación y plantearse "algo más allá” del modelo, que ha obnubilado a unos y enceguecido a otros y que hoy pareciera acercarse a su fin.

\section{El itinerario de cambio en democracia y el debate acerca de los resultados alcanzados}

Una de las primeras tareas del Gobierno democrático, en la década de los 90, implicó detener el proceso de reducción del tamaño del Estado y dio comienzo a una modernización en su accionar, que fundamentalmente buscaba regular muchos de los cambios que ya se habían impulsado en materia económica, a saber: disciplina fiscal, control del gasto, manejo de tipo de cambio, liberalización del sistema financiero, apertura a la inversión extranjera, privatización de empresas públicas, y las reformas al Estado, siendo estas últimas posibles de analizar considerando las tres perspectivas analíticas que coexisten respecto de la temática: la administrativa, los cambios institucionales y la reforma democrática (Tomassini; Armijo, 2002).

Específicamente la reconstrucción del sistema educativo público fue asumida desde los inicios del proceso democrático como una tarea que no podría volverse atrás, en términos de algunos aspectos ya consolidados, elementos identificados mucho tiempo después por la OCDE, al señalar "El gobierno de la transición a la democracia tomó la decisión estratégica de no revertir el proceso de municipalización ni cambiar el modelo y mecanismos de financiamiento establecidos en 1981" (OCDE, 2004, p.20). Con ello, las autoridades del nuevo Gobierno sabían que no estaban respondiendo a las expectativas de los docentes y, de alguna forma, validando los cambios generados en un régimen autoritario: "Tratar de reestructurar el sistema de financiamiento escolar en Chile produciría una fractura en el frágil equilibrio entre la izquierda y la derecha que formó parte implícita del acuerdo que reestableció el gobierno democrático." (2004, p.106).

Compartiendo los considerandos al respecto, son atendibles por cuando el modelo y mecanismos de operación financiera eran 'instrumentos de política educativa' tanto o más eficientes que las nuevas orientaciones políticas pro-reforma educativa. En consecuencia, hacer operar una reforma educativa sobre un sistema de financiamiento asentado en otros principios, algunos incluso contradictorios, es una tarea que agregó complejidad al diseño y - ciertamente - también ha incidido sobre los resultados alcanzados.

El mismo informe de la OCDE señala que la reforma educativa chilena de 1995 combina

... dos políticas de reforma coexistentes pero ideológicamente en conflicto. Una es la noción de que los mercados educacionales, con competencia entre escuelas, un alto grado de elección de los establecimientos educacionales por parte de los padres y una administración privada de dichos establecimientos proporcionan la mejor esperanza de eficiencia educacional y el rendimiento escolar más alto posible. La otra es la noción de que el gobierno central debe intervenir 
en el sistema educacional con una clara visión de lo que constituye una buena educación y cómo lograrla para asegurar que los estudiantes tengan la mayor oportunidad de aprender. (2004, p.289).

Esta fue la dinámica en el desarrollo del sistema educativo en estos años y hasta la fecha y, como se señaló en la introducción al trabajo, muchos pensaron que se podrían articular, reduciendo las diferencias y por esta vía solucionar los problemas del sistema educacional.

En el periodo en análisis se aplicaron diversas estrategias políticas en el accionar del Ministerio. El primer período está marcado fundamentalmente por metas reivindicativas y el reposicionamiento de la educación como un sector relevante del hacer del país. En razón de ello, destacan los esfuerzos por incrementar los recursos públicos al sistema, mejorar las condiciones de trabajo y remuneraciones del personal docente y no docente, y comenzar un proceso de mejoría mediante proyectos específicos del equipamiento e infraestructura escolar apoyados con recursos del Banco Mundial.

Gran parte (sino todas) de las iniciativas impulsadas son vía programas o proyectos y se instalan como tal en la orgánica ministerial, es decir, no forman parte de las tareas regulares del Ministerio, estrategia empleada para que éstas no fuesen absorbidas por la cultura organizacional vigente. Los primeros programas son de apoyo a las escuelas con más bajos resultados escolares (conocido como P900), y luego el impulso de un programa de Mejoramiento de Calidad y Equidad (MECE), que inicialmente fue para el nivel básico urbano, extendiéndose luego al lo rural y, posteriormente, a la enseñanza secundaria y superior. Las políticas, en este ámbito, son tipificadas como de calidad y equidad de la educación; de cambios incrementales, abiertas a la iniciativa de actores y del entorno, orientadas a la escuela como eje de acción (García - Huidobro; Cox, 1999, p.27-28). Este tema, la escuela como centro de la acción, corresponde a una visión estratégica que el Ministerio adopta además en lo programático, como una forma de romper la inadecuación del enfoque matricial 'Ministerio-Municipios', que no permite al primero (Ministerio) un adecuado control del hacer escolar más allá de algunas dimensiones importantes de carácter formal y claramente insuficientes para responsabilizarse del proceso pedagógico, como se le demanda al Ministerio, sin tener un soporte legal consistente con esta misión.

La estrategia de creación de programas para cumplir tareas específicas continúa hasta el año 2000. Aún cuando la Reforma Educativa es lanzada públicamente a mediados de la década de los 90, continúa esta política generando un conjunto de tensiones y de problemas que no siempre pudieron ser resueltas

Ausencia de un discurso unitario de la reforma que integre en un relato coherente y significativo el conjunto vasto de las distintas iniciativas y logre conectarlo con los temas de la tradición docente como: educación pública como función crucial del estado, la educación como actividad esencialmente moral -y secundariamente instrumental - rol central y de liderazgo del MINEDUC, solidaridad y no competencia, participación y no tecnocracia (García-Huidobro; Cox,1999. p. 44).

En definitiva, la reforma no articula debidamente el campo de las iniciativas y se implementa un sistema de respuesta -aunque no se quiera - en la lógica neoliberal: cada problema genera una respuesta específica o focalizada, en vez de diseñar una estrategia flexible y articuladora.

La Reforma Educativa impulsada en Chile planteó como eje central la transformación curricular en todos sus niveles. Ella se sustenta en un enfoque constructivista - similar al empleado en la reforma española ${ }^{8}$ - a la cual se sumó una línea de refuerzo de la profesionalización docente, que incluye aumento sistemático de remuneraciones, incorporación de incentivos, pasantías en el exterior, perfeccionamiento fundamental, cambios en la

${ }^{8}$ Cuando se señala "similar" es precisamente para establecer su proximidad. Sería un reduccionismo plantear que es la copia de la reforma española en este plano. Algunas de sus principales diferencias radican en que la reforma española tuvo un período inicial mucho más prolongado que la chilena y otra, muy importante, es que la do que la chilena y otra, muy importante, es que la
reforma española se centra y refuerza el rol de la escuela pública y con una profesionalización docente mucho más conciente que en el caso chileno. 
formación inicial y premios de excelencia. También se agrega una línea de implementación de la jornada escolar completa en los establecimientos escolares del país (aún vigente). Programas de mejoramiento de la calidad y equidad, y de apoyo de iniciativas focalizadas - que incluye el P-900, el ahora Programa Enlaces de informática educativa, el Proyecto Montegrande de liceos de anticipación y otras iniciativas - se fueron desarrollando con el avance de la reforma y, ciertamente, la línea de reforma curricular fue dirigida a construir y aplicar un nuevo marco curricular mínimo (GarcíaHuidobro; Cox, 1999, p.25).

Los resultados registrados a partir del año 1995 y hasta el 2006 (en que hay cifras comparativas) en los indicadores clásicos del sistema educativo se desplazaron positivamente. La cobertura de la educación básica subió del 92 al 99\%, y en la enseñanza media del 78 al 89\%; la tasa de deserción en básica se redujo del 4 al 1.7\% y del 12,6 al 6.0\% en enseñanza media; de igual forma, la tasa de alumnos aprobados en enseñanza básica subió del 92,3 a, 96\% y en la media del 81.5 al 90\% (MINEDUC, 2007). Este proceso también implicó un mejoramiento de la cobertura de la educación preescolar, superando el 33\% de la población incorporada a una modalidad de enseñanza, aunque con una claro sesgo socioeconómico (Herrera; Bellei, 2002). Paralelamente, la tasa bruta de participación en la educación superior sube de 28 a 37\% (Brunner; Elacqua, 2003, p.87; ÍNDICES, 2007).

Sin embargo, el impacto de los resultados reseñados sobre los aprendizajes de los alumnos en el sistema escolar no tiene la progresión esperada respecto de los insumos incorporados. Una cifra es elocuente, desde el año 1999 hasta el 2007 (MINEDUC, 2008) incluido, los rendimientos medidos en las Pruebas SIMCE son equivalentes o similares, esto es, se encuentran estancados.

La evidencia aportada por la comparación de los resultados SIMCE a lo largo de los 90 puede ser resumida en cuatro constataciones. Primero, que hay una tendencia consistente aunque leve, de incremento en los promedios nacionales en la primera mitad de la década, reduciendo así levemente las diferencias de logros entre establecimientos municipales y particulares pagados. Esta se estancó de 1996 a 2002 y la diferencia aumentó. Segundo, que la distribución social de los aprendizajes exhibe una distribución altamente estratificada e inequitativa, similar a la de 1990. Tercero, que las mejoras en rendimiento son mayores que las del promedio en el caso de las escuelas básicas que han sido objeto de programas focalizados como el P-900 o el Programa rural, lo que ha significado disminución de las brechas de rendimiento entre este alumnado-el más pobre - y el resto del país. Por último, que las diferencias de logros en el aprendizaje entre las distintas dependencias del sistema subvencionado (Municipal y Particular) son mínimas y no siempre favorables a la educación privada, cuando se comparan grupos socioeconómicos homogéneos (OCDE, 2004, p.39-40).

La síntesis expuesta en el informe de la OCDE permite formarse un panorama cierto del problema, pero es relevante hacer las siguientes puntualizaciones. La educación nacional avanzó lentamente hasta estancarse, siendo más relevante que el factor dependencia del establecimiento (si es público o privado) el factor socioeconómico de la familia, lo que confirma que la sociedad chilena es la segunda más desigual de América latina tras Brasil, y es una de las más desiguales del mundo (PNUD, 2003; Brunner; Elacqua, 2003). En consecuencia, el principal factor explicativo en los resultados escolares son los antecedentes socioeconómicos, variable que ha sido y sigue siendo dominante al extremo, que relega otras como dependencia - con aportes marginales (Donoso; Hawes, 2002). Adicionalmente, esta relación evidencia que la educación tiene un papel limitado en la reducción de la equidad y desigualdad. Lo que se refuerza, en segundo lugar, pues el estancamiento de los resultados responde también a la mantención, sino aumento, de la brecha de inequidad en la distribución del ingreso en Chile, lo cual si bien ha implicado cierta reducción de la pobreza, la distancia con los de mayores ingresos se incrementa (MIDEPLAN/ MINEDUC, 2004). Tercero, el sistema educacional chileno ha sido capaz de reintegrar a un conjunto de estudiantes que estaban fuera del sistema, que, en su gran mayoría, responden a los sectores de 
mayor vulnerabilidad social, sin bajar sus rendimientos, aspecto que no es menor, pero que no ha sido del todo destacado, y aparece más como excusa ante quienes critican que no se avanza a gran velocidad, que como elemento explicativo real.

De hecho, son los resultados escolares del SIMCE como de las pruebas de comparación internacional TIMMS - incluyendo los resultados para el año 2003 - y PISA del 2006 los que detonan el fin de esta etapa y el inicio de una 'crisis no resuelta hasta la fecha'.

Estos estudios revelaron que el sistema escolar estaba obteniendo bajos logros en comparación con los competitivos estándares de aprendizaje en el mundo. [...] mientras las bases del nuevo sistema escolar estaban ahora implementadas, las experiencias de aprendizaje estaban lejos de lo que requiere una sociedad crecientemente integrada en un mundo globalizado y aún más exigente en términos de conocimiento y habilidades de las personas y organizaciones (OCDE, 2004, p.35).

Dehecho, los resultados de las comparaciones internacionales ratifican dos hipótesis. La primera es que los logros de Chile no son buenos en ninguna estructura comparativa, es decir que los establecimientos escolares de elite social y económica del país no alcanzan logros comparables con los de sus pares de otras latitudes (confirmado incluso con la medición PISA del año 2006). Ello implica que el problema en Chile tiene una dimensión estructural importante y que los establecimientos particulares, comparados con sus pares, están significativamente más atrás. En consecuencia, privatizar, no parece ser un camino a seguir, más aún si sus precios promedio (valor colegiatura) más que triplican el valor de la subvención, no siendo sus resultados acordes a este diferencial de inversión. La segunda hipótesis en consideración es que, en las pruebas internacionales, los países con mayor desigualdad social reproducen, en parte importante, su mayor desigualdad educativa (García-Huidobro; Bellei, 2003, p.46).

Los intentos por revertir el estancamiento de los resultados educacionales, ya percibidos con fuerza el año 2002, reorientan el accionar del
Ministerio para 'llevar la reforma a la sala de clase’:

La respuesta de la política a este nuevo desafío está basada en dos pilares fundamentales: Primero una campaña para mejorar las habilidades de lectura, escritura y matemática de kinder a cuarto básico, lo que incluye el rediseño del curriculum de 1996 para los primeros cuatro cursos, cambiando las políticas de capacitación de los profesores, y haciendo esfuerzos especiales para obtener el apoyo de los padres. Segundo [...] el Ministerio de Educación se ha comprometido con el aseguramiento de calidad de los resultados, aplicando (entre otras medidas) la evaluación docente y los requisitos de desempeño específicos (OCDE, 2004, p.35).

Los resultados expuestos en esta sección muestran claramente que la calidad de la educación es la temática pedagógicamente dominante de una reforma que ha cumplido un ciclo importante en materia de recuperar para el país - aunque experiencialmente sea irrecuperable para las personas - los estándares tradicionales de capital educativo, quedando al debe la tarea - no sólo para la reforma, sino para el país - de mejorar significativamente en este ámbito. También queda en evidencia que esta temática de política educativa-la calidad - se asienta, en el caso chileno, sobre una base social extraordinariamente compleja, de desigualdad que se manifiesta en el ámbito social, económico, cultural y educativo. Aunque su tratamiento inicial correspondería a una política social, lo cierto es que se encuentra en el límite entre ambas, en razón fundamentalmente del rol que la educación cumple en este plano.

En razón de lo señalado, podríamos decir que la gran desigualdad de capital económico, social, educacional y cultural es la que está limitando la actual estrategia de desarrollo educativo implantada, requiriendo nuevas opciones para virar significativamente el status quaestionis de la reforma chilena.

En este marco, el 'aceptado' estancamiento de los resultados en materia de aprendizaje se ha transformado en el eje de un problema mayor cuya resolución podría precipitar una nueva reforma de más intensidad en las cuestiones hoy medulares, procesos que podrían ser vistos como 
contrarreforma, según se le defina. Por un lado, los resultados actuales son explicados sólidamente por la lógica de la causalidad estructural, la cual posterga toda opción de mejoría substantiva de los logros educacionales a un cuadro muy diferente del actual en materia de relaciones sociales del país. Por otro lado, quienes comparten los postulados del sistema de vales (vouchers) sostienen que se trata de un tema de eficiencia y de gestión del uso de los recursos, más que de diferencias sociales irremontables para el sistema educacional.

La discusión al respecto tiene los siguientes acentos, algunos autores (Aedo, 1988; Aedo; LarraÑaga, 1995) señalaban que los establecimientos particulares con subsidio tenían mejores resultados que los establecimientos municipales. Posteriormente, Mizala y Romaguera (2000, 2003, 2004), controlando esta relación por el capital humano inicial, dan cuenta que no había diferencias significativas en los resultados. A esta línea también se suma Tokman (2001). Un grupo de autores, Sapelli y Vial (2002), Gallego (2002), encuentran que hay diferencias que debidamente controladas son favorables a los establecimientos particulares subvencionados. Por otra parte, Donoso y Hawes (2002) señalan que el factor clave es el capital social y económico familiar, y Hsieh y Urquiola (2002) amplían esta explicación señalando que los resultados se explican finalmente por el traslado de los menos pobres a los establecimientos particulares con subvención del Estado.

Adicionalmente, es importante comprender que el mejoramiento de los indicadores de eficiencia interna no implica necesariamente un incremento de la calidad de los aprendizajes, como de hecho se puede deducir de los registros de las pruebas nacionales e internacionales para la presente década. Ello ha dado origen a un nuevo proceso de exclusión social, que cambia los parámetros anteriores establecidos del acceso o no acceso a la educación o del haber completado o no determinados ciclos o niveles. El nuevo proceso de exclusión implica logros formales equivalentes pero diferenciales de calidad muy desiguales. Esto se traduce en que existen segmentos de mala calidad de la educación, asociados también a precarias condiciones de vida, que generan un círculo de mala calidad que incluso puede implicar a la educación superior también (Donoso; Schiefelbein, 2007; Donoso; Cancino, 2007). Estas nuevas formas de exclusión social reproducen la desigualdad intergeneracional sólo que el punto inicial de educación formal es más alto, pero los resultados son los mismos.

Los estudiantes que se inician en los círculos de mala calidad de educación primaria lo continúan en la educación secundaria y, finalmente, muchos de ellos acceden a la educación superior de mala calidad. Por ende, no logran dar el salto generacional que la educación promete en su lectura más optimista del Capital Humano. Lo inverso también se confirma: los resultados educacionales ratifican que a quienes tienen más alta dotación inicial de capital social y cultural alcanzan mejores resultados y, por esta vía, se mantienen las diferencias sociales y, de esta forma, se reproduce y perpetúa la desigualdad, sólo que con nuevos rostros y nuevas dinámicas, pero con un credencialismo formal superior, aunque en los hechos estas "credenciales" tienen menos valor.

En consecuencia, el sustrato social sobre el cual ha operado el sistema educacional ha sido una sociedad altamente desigual, no sólo en materia de ingresos económicos, sino de calidad de vida; se trata de una desigualdad severa que conlleva una profunda injusticia (Rawls, 1986).

El problema de fondo es la concepción de un Estado que, en su racional último de funcionamiento, es subsidiario, por ende no cuenta con los mecanismos legales ni financieros para implementar todas sus acciones y responder por ellas sin negarse a si mismo como Estado. Es decir, requeriría renunciar su definición para asumir este rol. Esto se expresa en la falta de coherencia entre las políticas y los mecanismos de implementación, tal como fue expresado previamente, como también en los problemas de voluntad política para operar, con una lógica que no sea "de mercado", aquellos ámbitos de 'lo público' que no se ajustan a los criterios de la racionalidad privada, es decir, al apli- 
car "los esquema de competitividad de mercado" al sector público, se privatiza su operación, de manera que termina respondiendo a ese racional.

\section{LA SOLUCIÓN RESIDE EN EL DISEÑO DE UN NUEVOESTADO}

\section{La adaptación como propuesta de cambio para el Estado}

La hipótesis de trabajo que sustenta la ponencia sostiene que la crisis del diseño político del sistema educativo chileno se ha tornado estratégica, pues, durante casi dos décadas, los gobiernos democráticos han buscado compatibilizar dos racionales asentados en fundamentos y criterios de operación que han mostrado su contradicción, a saber, el de Estado subsidiario versus el de Estado solidario, proceso impulsado tras el retorno a la democracia, cuyo racional ha consistido en extremar las semejanzas y minimizar las diferencias entre ambas perspectivas. Los resultados reseñados evidencian que el camino a seguir en la actualidad parece cada vez más estrecho y difuso, próximo a agotarse, requiriendo, para la búsqueda de solución, analizar nuevos diseños políticos para el sistema educacional chileno, al amparo posiblemente de la discusión de un nuevo rol del Estado.

A la fecha las orientaciones de cambio del Estado han seguido tres vertientes fundamentalmente técnicas, cuyo desenvolvimiento no entra en cuestionamiento con el rol subsidiario del Estado. Una de las cuales, la denominada de 'gerencia pública', busca impulsar, en la instituciones del Estado, la visión del desempeño, con énfasis en los resultados, su medición, la asignación de estímulos, orientada al servicio al usuario, que busca nuevos diseños institucionales que cambien la cultura de 'entidad monopólica' a diseños más flexibles y horizontales, donde la rendición de cuentas, de descentralización y desconcentración de sus funciones sean prácticas regulares. La reforma gerencial del Estado, que proviene de la experiencia cultural anglosajona, ha sido adoptada en el Chile aún como algo formal, aunque en la actualidad se han dado pasos ciertos en esa dirección.

Esta tarea ha sido complicada, pues ha debido ordenar situaciones que estaban operando desregladamente y que eran claramente asimétricas entre oferentes y demandantes, lo que implica "normar" un mercado muy desprotegido para los beneficiarios. Ello fue posible observar con mayor facilidad en la administración de servicios públicos monopólicos, como agua y electricidad y, fundamentalmente, con el sistema de pensiones, de salud y también de educación, donde el mercado privado es muy poderoso y se ha opuesto tenazmente a perder parte substantiva de sus privilegios.

La segunda perspectiva analítica se refiere al cambio institucional, producto de las nuevas demandas sociales de grupos emergentes, desprotegidos o bien insatisfechos, impulsados además por requerimientos tecnológicos que presionan por una nueva institucionalidad. Se trata de un nuevo equilibrio entre el mercado, el Estado y la sociedad civil que, a partir del tácito e incuestionable peso del mercado, busca revisar y redireccionar sus políticas públicas, sin transgredir los equilibrios macroeconómicos fundamentales, cuyo aprendizaje de este principio operativo fue pagado por América Latina a un alto precio.

Las Reformas de tipo gerencial buscan modernizar la gestión de los servicios públicos encargados de ejecutar las políticas gubernamentales, en tanto que el rediseño de las instituciones del Estado tiene por objeto habilitarlo para formular más eficientemente esas políticas (Tomassini; Armijo, 2002, p.26).

Sin embargo, en el caso chileno, el fortalecimiento de la capacidad del Estado para satisfacer por si mismo la demanda ciudadana es una temática aún incipientemente discutida, sea porque las leyes 'de amarre' de la época dictatorial aún imponen restricciones al hacer de un Estado más empresarial, también porque la validación de la visión neoliberal de la sociedad y del mercado han desacreditado el accionar directo del Estado como ejecutor de políticas, amén de los problemas ciertos 
de eficiencia que ha mostrado, y asimismo porque, al interior de la coalición política, el neoliberalismo tiene también importante arraigo.

La tercera perspectiva implica asumir una visión de gobernabilidad sustentada en la democratización del Estado, lo que debe traducirse en capacidad para identificar e interpretar adecuadamente las demandas ciudadanas y traducirlas en políticas según las prioridades alcanzadas en las agendas de debate. Esto significa incorporar las visiones anteriores de reformas al Estado para que éste se desempeñe no sólo como un agente regulador sino también, de igual manera, como un actor direccionador de las políticas según su capacidad para interpretar y traducir demandas y necesidades en propuestas de acciones. Esta situación implica para el Estado ampliar su capacidad de integrar e integrarse a la sociedad civil. Esta visión no siempre es comprendida en su sentido biunívoco, sino esencialmente se la entiende como el considerar los problemas de los usuarios para buscar soluciones, pero el rol de participación activa de la sociedad civil en la definición de políticas es mucho menos practicado, aunque se le entiende como una condición clave para la adopción de decisiones adecuadas y oportunas, siendo constitutivo de la concepción de gobernanza hoy mayoritariamente aceptada.

\section{Desde el Estado: su rol en la provisión de educación}

Asumiendo que, en la concepción actual del rol del Estado, su principal tarea es la formulación de las políticas públicas con la finalidad de orientar y regular el comportamiento de los distintos agentes económicos y sociales, toda vez que su rol ejecutor está en segundo orden, dada la plena competencia de privados o del mercado globalizado, su tarea se compatibiliza en velar por el interés público y de los diversos grupos sociales, según su grado de vulnerabilidad. En razón de lo señalado, las implicancias de este considerando son estratégicas, pues se sabe que de la calidad y oportunidad de las políticas públicas dependerá, en grado importante, la gobernabilidad del país. ${ }^{9}$

La crisis del Estado también tiene una vertiente diferente de análisis. En el formato del Estado chileno, las competencias esenciales de esta tarea residen en el Estado central. Los Gobiernos subnacionales no desempeñan todavía un papel relevante que no sea aquel de cumplir con algunas funciones descentralizadas o desconcentradas, representación de autoridades u otras, entre las que pueden estar las de hacer cumplir normativas nacionales (replicar la función del Ministerio en el ámbito subnacional en estas tareas), obtener información y entregarla ordenada para su procesamiento central, quedando claro que el tema del diseño y estructuración de las políticas es una tarea que esencialmente le compete al ámbito central.

El accionar del gobierno nacional y por extensión de los gobiernos subnacionales en el campo educacional esta referido al cumplimiento de una tarea central y común del país, que es incluir a toda la población en edad escolar en el sistema educativo, labor que puede ser una tarea rutinaria como un desafío de proporciones según el nivel de desarrollo alcanzado. De este primer quehacer, se desprenden dos imperativos claves de gestión: el primero es proveer una educación de calidad para todos y, paralelamente, sentar las bases de construcción y mantenimiento de la sociedad, proceso de socialización que implica legitimar la diversidad a fin de fortalecer la ciudadanía y la democracia.

Como fue señalado, el problema reside en que la calidad de la educación presenta estándares muy desiguales entre los diversos estratos sociales, de manera que, aunque teniendo semejanzas formales, en el fondo, las diferencias son claves y responden a una acumulación de "ventajas o de desventajas", según sea el caso, que finalmente concluyen en una reproducción de la desigualdad social intergeneracional, sólo que debidamente legitimada. Quienes

${ }^{9}$ Entendida como la capacidad del Gobierno para articular, ordenar y responder en forma armónica las distintas demandas ciudadanas por desarrollo social y económico, participación y equidad, de forma que pueda continuar gobernando, se entiende, bajo principios democráticos si esta es la tipificación de la gobernabilidad. 
asisten en la enseñanza primaria a establecimientos de baja calidad educacional continúan una enseñanza media de baja calidad e incluso pueden ingresar a al educación superior a opciones de escasa inserción laboral. Este círculo reproductivo de la desigualdad es la nueva forma como se presenta el problema, que ya no es de "excluidos e incluidos", sino de nuevas formas de inclusión o exclusión más complejas, pero igualmente eficientes para mantener las diferencias intergeneracionales.

Los aspectos reseñados evidencian que, tras este proceso, hay un diseño político de un sistema social que se muestra incapaz de solucionar los problemas endémicos de desigualdad social que ha caracterizado a la sociedad chilena al menos desde el siglo XX en adelante, mostrando con ello una crisis profunda no solamente del sistema educacional sino del sistema social y económico, lo que demanda para su cambio bajo un formato sistémico, diferente ciertamente al actual y que no se vislumbra aún como factible.

\section{EL DEBATE ENTRE POLIITICAS DE ESTADO Y POLÍTICAS DE MERCADO}

La severa crisis de la educación chilena no solamente involucra sus resultados de aprendizaje; su profundidad, persistencia y amplitud dan cuenta del involucramiento de la mayor parte de las dimensiones del sistema. La concepción subsidiaria del Estado, alzada por el neoliberalismo, no permite el diseño de la institucionalidad articulada del sector que relacione funcional y equilibradamente ${ }^{10} \mathrm{a}$ los responsables nacionales

${ }^{10}$ El equilibrio proviene de la adecuada aplicación del principio de responsabilidad-autoridad. Sin embargo, el Ministerio de Educación asume algunas responsabilidades para las que no tiene autoridad, dependiendo para ello de la voluntad de los empresarios. Un ejemplo se vivió en el perfeccionamiento para la reforma. En oportunidades empresarios enviaron a capacitarse a profesores para cursos en que posteriormente no se desempeñaron, sin que el Ministerio pudiera intervenir. Asimismo, algunos empresarios públicos deben responsabilizarse de aspectos para los que no tiene autoridad. Por ejemplo, la ley define concurso para directivos - docentes, no existiendo los fondos para ello, obligando a los municipios a aumentar su gasto, ya que no tiene la autoridad para negarse al concurso. de la educación (Ministerio) con los responsables locales: municipios y empresarios privados. Se trata de un diseño matricial que no ajusta. En lo operacional la matriz "Ministerio-Municipio" no tiene mecanismos de compatibilidad, derivando en contradicciones de proporciones. Consistente con esta visión del Estado, el Ministerio de Educación no ha podido generar alianzas estratégicas con socios estables.

En segunda instancia, no existe una definición básica - aceptada mayoritariamente de lo que se entiende por educación (más allá del documento 'Marco para la Buena Enseñanza'), que confiera coherencia al conjunto del sistema. Ello facilita realizar cambios parciales sin comprender su impacto en el sistema, permitiendo que las políticas referidas al mejoramiento de calidad y eficiencia sean más resistentes al cambio (regímenes de contratación, remuneraciones e incentivos salariales de los maestros) y otras más adaptables (expansión y crecimiento de la matrícula). Estas relaciones responden al "hábito cultural" de enfocar el tema como si el centro fuesen los docentes y sus gremios. Ello facilita dividir el fenómeno educativo (siendo, para algunos, indivisible) de manera que no se perciban los impactos entre los diversos componentes, lo que ha provocado la instalación de iniciativas sectoriales que - en oportunidades no son consistentes con las políticas generales.

La generación de una Política de Estado es consistente con el diagnóstico precedente. Sin embargo, esa propuesta implica un Estado políticamente activo como regulador y organizador del sistema educacional en todo su sentido, y que detente las atribuciones para cumplir su tarea. Ello es un contrasentido en un esquema de mercado o un modelo deEstado Subsidiario; en consecuencia, en alguna medida, se exige algo que está fuera del racional que no se quiere o puede cambiar.

El Estado ha manifestado un compromiso medido en los hechos con una educación de calidad. La sociedad, en forma mayoritaria, reclama para el sector educación “otro Estado". Si embargo, la demanda de una educación de calidad no está del todo asentada en la sociedad civil, en 
términos de un canal unívoco de expresión y acción al respecto. Tampoco está plenamente enraizada en los gremios docentes -donde se mezcla con reivindicaciones salariales -, ni en los de estudiantes y padres, que carecen de la competencia para traducirles de demandas sentidas a requerimientos técnicos. Ello es funcional a una visión tecnocrática de la política. Sin embargo, la esperanza de mejoría se confronta con una visión de mercado que no puede destruirse a si mismo para solucionar sus graves problemas. La posibilidad de generar consensos es compleja: la educación es un campo de fuertes intereses ideológicos, también de clase y, por ende, de poder.

El Estado chileno tiene una baja cuota de poder en este escenario. Su legitimación está en duda en términos de ofrecer respuesta a las necesidades y garantizar a los ciudadanos un sistema educativo de calidad: algo que, en la actualidad, es ampliamente demandado por la sociedad e insatisfactoriamente cumplido en la educación pública, fenómeno que, en el formato de mercado, parece irresoluble, salvo que opte - al menos inicialmente - por su auto destrucción.

(Recebido para publicação em julho de 2009) (Aceito em setembro de 2009)

\section{REFERÊNCIAS}

AEDO, C.; LARRAÑAGA, O. Educación privada versus educación pública en Chile: calidad y sesgo de selección. Santiago, Chile: Programa de Postgrado en Economía ILADES/Georgetown University,1995.

Diferencias sobre escuelas y rendimiento estudiantil en Chile. En: _. La organización marca la diferencia: Educación y salud en América Latina. Santiago, Chile: Programa de Postgrado en Economía ILADES Georgetown University, 1998.

ALMONACID, C.; LUZÓN, A.; TORRES, M. Cuasi mercado educacional en Chile: el discurso de los tomadores de decisión. Archivos Analíticos de Políticas Educativas, v.16 n.8, 2008. Disponible en: http://epaa.asu.edu/epaa/v16n8/ Acceso en: mayo 2008

BRUNNER, J.J.; G. ELACQUA, G. Capital humano en Chile. Santiago, Chile: Universidad Adolfo Ibáñez. 2003

CARO, J. El contexto histórico de la descentralización.
En: VERGARA, J.; VON BAER, E. En la frontera del desarrollo endógeno. Temuco, Chile: Ediciones Universidad de la Frontera, CIDER, 2004. p. 413-446.

CANCINO, Víctor; DONOSO, Sebastián. El programa de Informática Educativa de la Reforma Educativa Chilena: análisis Crítico. Revista Interamericana de Educación. Santiago, Chile, OEI, n. 36, 2004

COMITÉ TÉCNICO ASESOR DEL DIÁLOGO NACIONAL DE MODERNIZACIÓN DE LA EDUCACIÓN CHILENA. LOS desafíos de la educación chilena frente al Siglo XXI. Santiago, Chile: Presidencia de la República, Ministerio de Educación, 1994.

CONSEJO ASESOR PRESIDENCIAL PARA LA CALIDAD DE LA EDUCACIÓN. Informe Final. Dic.2006, Santiago, Chile. Disponible en:<www.presidencia.cl. Acceso en: dic. 2006.

DIPRES. Presupuesto del Ministerio de Educación. Santiago, Chile: Ministerio de Hacienda, Dirección de Presupuesto, 2003.

Ejecución Presupuestaria Ministerio de Educación. Santiago, Chile: Ministerio de Hacienda, Dirección de Presupuesto, 2004.

DIRECTIVAS presidenciales para la educación. Santiago, Chile: Presidencia de la República, 1980.

DONOSO, S.; HAWES, G. Eficiencia escolar y diferencias socioeconómicas: a propósito de los resultados de las prueba de medición de la Calidad de la Educación en Chile. Educação e Pesquisa, São Paulo, v. 28, n. 2, p. 25-40, 2002.

; CANCINO, V. Caracterización socioeconómica de los estudiantes de educación superior en Chile. Calidad en la Educación. Mexico,DF, IRISIE, n. 26, p. 206-244, 2007.

; SCHIEFELBEIN, E. El Sistema de Selección de Estudiantes a las Universidades Chilenas: Un mecanismo eficiente de reproducción y validación de la desigualdad intergeneracional. Proposal submited CONFERENCE INTRA AND INTERGENERATIONAL TRANSMISION OF INEQUALITY. Mexico: El Colegio de México Feb. 28-29 2008.

SCHMAL, R. Introducción a la economía de la educación: nociones fundamentales. Talca, Chile: Universidad de Talca, 2008.

GALLEGO, F. Competencia y resultados educativos: teoría y evidencia para Chile. Cuadernos de Economía, Santiago, Chile, n.118, p.309-352, 2002.

GARCÍA HUIDOBRO, J. E. Parte I. Visión de Conjunto Capítulo 1. La Reforma Educativa Chilena 1990 -1998. En: GARCIA-HUIDOBRO, J.E. (Ed.) La reforma educativa chilena. Madrid: Ed. Popular, 1999. p.7-50.

; COX, C. Capítulo I: La Reforma Educacional ChiTena 1990 -1998. Visión de Conjunto. En: GARCIA HUIDOBRO (Ed.) La reforma educacional Chilena. Madrid: Editorial Popular, 1999. p.7-46.

; BELLEI, C. Desigualdad educativa en Chile. Santiago, Chile: Universidad Alberto Hurtado, 2003, 62 p.

GONZÁLEZ, P. Estructura Institucional, recursos y gestión en el sistema escolar chileno. En: COX, C. (Ed.). Políticas educacionales en el cambio de siglo. La reforma del sistema escolar en Chile. Santiago, Chile: Editorial Universitaria, 2003. p.597-60.

. La igualdad educativa, el financiamiento vía subvenciones y la administración privada de la educación, elementos para la discusión. En: FORD, F. UNESCO UNICEF, U. Alberto Hurtado. Políticas educativas y equidad. Santiago, Chile: 2005. p. 247-270.

HSEIH, CH,: URQUIOLA, M. When school competes, how 
they compete? An assessment of Chile's nation wide school voucher program. Washington,D.C.: World Bank's Development Group, 2002.

HOXBY, C. Ideal Voucher. Chicago: University of Chicago Press, 2002.

ÍNDICES Estadísticas de la educación superior chilena. Santiago, Chile: Consejo Superior de Educación del Chile, 2008 .

JOFRÉ, G. Subvenciones en educación. Estudios Públicos. Centro de Estudios Públicos, Santiago, Chile, n. 32, p. 3155, 1988.

HABERMAS, J. Problemas de legitimación del capitalismo tardío. Buenos Aires: Amorrotyu, 1975.

MIDEPLAN/MINEDUC. Educación y pobreza. Resultados de la encuesta Casen 2003. Disponible en: www.mideplan.cl/ casen. Acceso en: 27 oct, 2004.

MINEDUC. Anuario Estadístico 2005. Santiago. Chile: Ministerio de Educación, 2006.

Anuario Estadístico 2006. Santiago, Chile: Ministerio de Educación, 2007.

Resultados de las Pruebas SIMCE 2007. Santiago de Chile: Ministerio de Educación, 2008.

MIZALA, A.; ROMAGUERA, P. Regulación, incentivos y remuneraciones de los profesores en Chile. En: COX, Cristián (Ed.) Políticas Educacionales en el Cambio ... Santiago, Chile: Piie, 2003. p. 519-568.

; . El Sistema Nacional de Evaluación del Desempeño docente (SNED) en Chile. En: BID- PREAL. Maestros en América Latina: nuevas perspectivas sobre su formación y desempeño. Santiago, Chile, 2004. p.389-407.
NEF, J. El concepto de Estado subsidiario y la educación como bien de mercado: un bosquejo de análisis político Revista Enfoques Educacionales, Santiago, Chile Departamento de Educación, Facultad de Ciencias Sociales, Universidad de Chile., v. 2, n. 2, 1999-2000.

OCDE. Chile. Revisión de las políticas Nacionales de Educación. Paris: Organización para la Cooperación y el Desarrollo; Santiago, Chile: Ministerio de Educación, 2004.

PNUD. Desarrollo humano en Chile 2002. Santiago, Chile: Programa de las Naciones Unidas, 2003.

RAWLS, J. Justicia como equidad. Madrid: Editorial Universitaria Tecnos, 1986.

ROJAS, P. Renumeraciones de los profesores en Chile. Estudios Públicos, Santiago, Chile, Centro de Estudios Públicos, n.71,p.122-175, 1998.

SAPELLI, C. Introducción: la economía de la educación y el Sistema Educativo Chileno. Cuadernos de Economia, Santiago, Chile, n. 118, p. 281-296, 2002.

;VIAL, B. The performance of private and public schools in the Chilean voucher system. Cuadernos de Economía, Santiago, Chile, n. 118, p. 423-52, 2002.

TOMASSINI, L.; M. ARMIJO, M. Reforma y modernización del Estado. Santiago, Chile: Lom Editores, 2002.

WEBER, M. El político y el científico. Madrid: Alianza Editorial, 1969. 


\section{THE CRISIS OF POLITICAL DESIGN OF THE CHILEAN SOCIAL SYSTEM: reproduction of intergenerational social inequality and new forms of social exclusion}

\section{Sebastián Donoso Díaz}

The political design of the Chilean social system has been unable to solve the endemic problems of social inequality that characterize it, showing thereby a deep crisis not only of the educational system but also of the social and economic system, which requires a systemic design different, of which there is no indication yet that it is feasible. Despite the effort expended, the educational results show a strong link with the social origin and economic status of families, i.e. the differences in initial social and cultural capital are not reduced, adjusted or reversed significantly by the regular educational system. The problem lies in the fact that education has very uneven standards among different social strata, so that, even with formal similarities, at bottom, the differences are key and respond to an accumulation of "advantages" and "disadvantages", according to each case, which finally result in reproduction of intergenerational social inequality, only duly legitimated.This reproductive circle of inequality is the new way by which the problem presents itself, no longer about "excluded and included, but about complex new forms of exclusion or inclusion, but equally effective at maintaining the inter-generational differences. The failure of the current design of the social and educational system entails viewing changes that require a change of magnitude in the design of the State, which is estimated to be complex, because, somehow, is to deny itself as a State, to give a step toward a new social pact.

KEYWORDS: social inequality, the Chilean educationa system, social and cultural capital.

\section{LA CRISE DE LA CONCEPTION POLITIQUE DU SYSTÈME SOCIAL CHILIEN: reproduction des inégalités sociales intergénérationnelles et nouvelles formes d'exclusion sociale}

\section{Sebastián Donoso Díaz}

La conception politique du système social chilien a été incapable de résoudre les problèmes endémiques d'inégalité sociale qui le caractérisent. Ceci démontre une crise profonde non seulement du système éducatif mais aussi du système économique et social qui requiert une conception systémique différente que rien ne laisse encore entrevoir. Malgré les efforts déployés, les résultats obtenus sur le plan éducatif mettent en évidence la relation importante qui existe entre l'origine économique et sociale, c'est-à-dire que : les différences de capital social et culturel initial ne diminuent ni ne sont corrigées ou inversées de manière significative par le système éducatif régulier. Le problème réside dans le fait que l'éducation présente des modèles extrêmement inégaux qui varient en fonction des couches sociales de telle sorte que même s'ils présentent des similitudes formelles, dans le fond les différences en sont la clé et répondent, selon les cas, à une série 'd'avantages et d'inconvénients' qui finissent par reproduire les inégalités sociales intergénérationnelles, mais dûment légitimées. Ce cercle de reproduction des inégalités est une nouvelle manière de présenter le problème qui ne traite plus 'd'exclus et d'inclus' mais de nouvelles formes complexes d'inclusion ou d'exclusion, tout aussi efficientes pour que les différences intergénérationnelles soient maintenues. Léchec de la conception actuelle du système éducatif et social suppose des changements capables d'exiger un changement radical de la conception de l'État, chose complexe vu que d'une certaine manière il s'agit de se nier soi-même en tant qu'État, afin de faire le pas vers un nouveau pacte social.

MOTS-CLÉS: inégalité sociale, système éducationnel chilien, capital social et culturel.

Sebastián Donoso Díaz - Doutor em Educação pela Universidade Academia de Humanismo Cristiano (Chile). Professor do Instituto de Investigação e Desenvolvimento Educacional da Universidade de Talca, Chile. Coordenador do Programa de Mestrado em Política e Gestão Educacional da Universidade de Talca. Integra o Núcleo de Pesquisa em Políticas Educionais do Centro de Investigação Avançadas em Educação (Financiado pelo Conselho de Investigação Científico e Tecnológico de Chile, desenvolvendo pesquisas na área de políticas educacionais e resultados escolares. Suas mais recentes publicações, são: DONOSO, Sebastian. El Financiamiento de la educación pública chilena vía subsidio: consecuencias críticas sobre el pol del Estado en educación. FINEDUCA. Revista de Financiamiento da Educaçao, v. 1. 2011. Revista electrónica, disponible en http://seer.ufrgs.br/fineduca; GARCÍA, Leidy; CERDA, Arcadio; DONOSO, Sebastián. Determinantes de la calidad docente: programa de asignación de excelencia pedagógica. INNOVAR. v. 21, n. 39, enero-abril de 2011; DONOSO, Sebastián; Arias, Oscar . Diferencias de escala en los sistema de educación pública en Chile. Ensaio, v.19, n.71, abril/junho, 2011; DONOSO, Andrés; DONOSO, Sebastián. Las discusiones educacionales en el Chile del Centenario. Estudios Pedagógicos, v.36, n.2, p.305-321, 2010. 